\title{
COMPUTABILITY OF THE EMERGENCY SERVICE SYSTEM DESIGN PROBLEM
}

The paper deals with emergency medical system design using methods of mathematical programming. The problem consists in optimal location of stations, where ambulance vehicles should be placed. Several possible objective functions are discussed and the relevant mathematical programming models are presented. The comparison of the solutions is reported based on the computational experiments in the conditions of the Slovak Republic.

\section{Introduction}

The medical emergency system design is a crucial task for each responsible designer due to the interaction of two opposite demands on the system performance. On the one hand the designer is forced not to exceed a given number of located facilities - ambulance vehicles and to solve a large facility location problem. On the other hand, he must ensure the accessibility of the service for potential patients. This accessibility is usually given by a fixed time limit, in which some ambulance vehicle should reach an arbitrary located potential patient [2], [12], [13].

This last demand is hard to meet, because of random travel time on a real road network. In addition, when an accident occurs, an ambulance starts its trip to the accident location to provide the service, which consists of first aid to causalities and their transportation to a hospital. Within this service, the facility (ambulance vehicle) cannot perform any service of other demands. It means that if some other accident occurs simultaneously in the area of this vehicle, then some other ambulance must serve it, or service of the later accident must be considerably postponed. This way, the service system works like a queuing system [11]. Under these circumstances, the access condition cannot be fulfilled absolutely, but only with some probability. Due to the impossibility to include means of the queuing theory into analytical models of the location problem, there are used various surrogate criteria such as an average or total travel time from the ambulance location to potential patients, which belong to the ambulance servicing area [6], [7], [8]. Another type of criterion (covering criterion) is that a maximal travel time from the nearest ambulance location to a customer should not exceed a given value. Designers face the above-mentioned ambulance occupation by using so called double coverage criterion, which is formulated so that the number of potential patients, which lie within a given time radius of two or more facilities should be maximal [2].
Usage of each of these criteria leads to a particular model of mathematical programming [5]. An exact method applied to the particular model has its specific demands for computational time and memory. In the next sections, we present an overview of these criteria, report about preliminary computational experiments and perform a comparison of them.

\section{The emergency service system design problem and quality criteria formalization}

Within the scope of this paper we confine ourselves to the problem, in which a medical emergency service system is designed. In contrast to the private service systems, the objective of this sort of public service system should stress equity of a "customer" in access to the provided service.

The emergency service system design belongs to the family of location problems [1], in which it must be decided on centre locations, where ambulance vehicles should be placed, because an effective satisfaction of the potential patient demands is possible only if the corresponding service provider concentrates its sources at several places of the served area and provides the service from these places only. The served area consists of dwelling places placed in nodes of a road network. These dwelling places form a finite set $J$. The number of inhabitants of dwelling place $j \in J$ will be denoted as $b_{j}$. The emergency service system design problem can be formulated as a decision about location of at most $p$ emergency centers at some places from a larger set $I$ of possible center locations so that the value of chosen criterion is minimal. The question, which must be answered first, is: "How to estimate the time of access to a customer?" Let $j$ be customer's location and $i$ be a centre of the service provider. Both the locations are nodes of a road network, which consists of links and nodes. Based on the link quality, each link belongs to a class from a finite classification

\footnotetext{
* Jaroslav Janacek, Ludmila Janosikova

Department of Transportation Networks, Faculty of Management and Informatics, University of Zilina, Slovakia,

E-mail: jaroslav.janacek@fri.uniza.sk
} 
system. In accordance to this system, an average speed is assigned to each link. This way, an estimation of the necessary traversing time for each link can be obtained from the link length and the average speed corresponding to the link class. Using this time instead of the link length, the accessibility time $t_{i j}$ can be enumerated as the time length of the shortest path in the network connecting $i$ and $j$. Time $t_{i j}(\boldsymbol{v})$ is a function of vector $\boldsymbol{v}=\left\langle v_{1}, v_{2}, \ldots ., v_{r}\right\rangle$ of the speeds, which corresponds to the particular link classes. Nevertheless, the average speeds are not constant, but they depend on weather, traffic volume and other dynamically changing conditions. Considering this condition variability, no system design ensures full satisfaction of the estimated time and each further developed criterion is enumerated in accordance to a given speed scenario $\boldsymbol{v}$. Let $i(\boldsymbol{v}, j)$ represent the located centre, which is the timenearest one to $j$ considering the link speeds given by $\boldsymbol{v}$. Further, let $I_{1} \subseteq I$ denotes the set of places, in which an ambulance vehicle is located.

After these preliminaries, we formulate the particular criteria. The first family of "allocation criteria" is represented by the total travel time from the ambulance location to potential patients. This criterion can be described by the following expression:

$$
\sum_{j \in J} b_{j} t_{i(v, j) j}(v)
$$

This criterion doesn't reflect equity of a "customer" in access to the provided services at all. The original requirement of the concerned public is that each inhabited place must be reachable within time $T^{\max }$ from at least one service centre from set $I_{1}$. The next criterion, which also belongs to the family of "allocation criteria" takes into account the size of affliction of potential patients, which are out of the time limit:

$$
\sum_{\substack{j \in J \\ t_{i(p, j), j}(\boldsymbol{v})>T^{\max }}} b_{j}\left(t_{i(v, j), j}(\boldsymbol{v})-T^{\max }\right)
$$

The second family of "covering criteria" [2] is represented by the criterion, which simply counts the potential patients, which are out of the time limit:

$$
\sum_{\substack{j \in J \\ t_{i(p, j) j}(v)>T^{\max }}} b_{j}
$$

The third family of "double coverage criteria" [2] is represented by the criterion, which counts the potential patients not covered at least from two ambulance locations. It is said that a patient is covered if the distance to the nearest ambulance station is less than a given limit $T^{\max }$. Let $s(\boldsymbol{v}, j)$ represent the second time-nearest station to $j$ considering the link speeds given by $\boldsymbol{v} ; s(\boldsymbol{v}, j)$ belongs to the set $I_{1} \subseteq I$ of places, in which an ambulance vehicle is located. The formulation of the last criterion can be as follows:

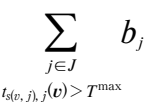

The expressions (1)-(4) are to be minimized subject to the constraint that the number of located facilities must not exceed the given number $p$.

The next generalization of these criteria may issue from observation of possible scenarios of the vehicle speeds. The family of the scenarios constitutes finite set $\boldsymbol{V}$ of possible speed vectors $v_{q}$, $q=1, \ldots, m$ and each scenario may be weighted by coefficient $h_{q}$. The weights can be set proportionally to the empirical frequencies or arbitrary else to reflect the necessity to keep the accessibility condition at a sensible level. The further generalization can be obtained by optimising a linear combination of criteria, where particular criteria are weighted according to their importance.

\section{Models and solving techniques for the emergency service system design problem}

The mathematical programming approach to the emergency system design comes out from the assumption that the ambulance vehicles are allowed to be located only at some places from the finite set $I$ of possible locations. The decision on placing or not placing an ambulance vehicle must be done for each candidate location $i \in I$. This decision can be modelled by the variable $y_{i}$, which takes the value 1 if a vehicle is placed at location $i$ and it takes the value 0 otherwise. The case, in which it is possible to place more than one vehicle at one location, can be rearranged to the considered zero-one decision problem by duplication or triplication of the relevant locations.

The emergency system design problem with the criterion (1) cannot be described only by the location variables $y_{i}$, due to the fact that the individual contribution to the objective function value depends on the distance between the customer and the nearest located ambulance. To be able to describe this sort of relations, we introduce zero-one variables $z_{i j}$ for each pair $\langle i, j\rangle$ of a possible location and a customer. Using these variables, the assignment of each customer to some ambulance location can be easily described. If we denote $c_{i j}=b_{j} t_{i j}(v)$, then the following model describes the emergency system design problem with the criterion (1).

$$
\begin{array}{ll}
\text { Minimize } \sum_{i \in I} \sum_{j \in J} c_{i j} z_{i j} & \\
\text { Subject to } \sum_{i \in I} z_{i j}=1 & \text { for } j \in J \\
z_{i j} \leq y_{i} & \text { for } i \in I, j \in J \\
\sum_{i \in I} y_{i} \leq p & \text { (8) } \\
y_{i} \in\{0,1\} & \text { for } i \in I \\
z_{i j} \in\{0,1\} & \text { for } i \in I, j \in J
\end{array}
$$

The expression (5) corresponds to the sum of the real access times multiplied by numbers of afflicted inhabitants. The constraints (6) ensure that each dwelling place (customer) is assigned to the 
exactly one of possible locations. The constraints (7) are so called binding constraints, which force the variable $y_{i}$ take the value 1 , whenever a customer is assigned to location $i$. The constraint (8) puts the limit $p$ on the number of located vehicles.

The model (5)-(10) describes also the emergency system design problem with the criterion (2). It is sufficient to denote $c_{i j}=b_{j}\left(t_{i j}(v)-T^{\max }\right)$, if $t_{i j}(v)>T^{\max }$ and $c_{i j}=0$ otherwise.

The problems connected with criterion (3) can be modelled using a set of the auxiliary zero-one variables $x_{j}$, which express by the values 1 or 0 , whether the demand of customer $j \in J$ is or is not satisfied. To be able to recognize, whether customer $j$ is or is not accessible from location $i$, we introduce zero-one constant $a_{i j}$ for each pair $\langle i, j\rangle \in I \times J$. The constant $a_{i j}$ is equal to 1 if and only if customer $j$ can be reached from location $i$ in the access time $T^{\max }$, i.e. $t_{i j}(v) \leq T^{\max }$. Otherwise, the constant $a_{i j}$ is equal to 0 . Then we can formulate the problem as:

$$
\begin{aligned}
& \text { Minimize } \sum_{j \in J} b_{j}\left(1-x_{j}\right) \\
& \text { Subject to } \sum_{i \in I} a_{i j} y_{i} \geq x_{j} \quad \text { for } j \in J \\
& \sum_{i \in I} y_{i} \leq p \\
& y_{i} \in\{0,1\} \\
& \text { for } i \in I \\
& x_{j} \in\{0,1\} \\
& \text { for } j \in J
\end{aligned}
$$

The objective function (11) gives the volume of uncovered demands. The constraints (12) ensure that the variables $x_{j}$ are allowed to take the value 1 , if and only if there is at least one ambulance vehicle located in the access time $T^{\max }$ from the customer location $j$. The constraint (13) puts the limit $p$ on the number of located vehicles [10].

The model (11)-(15) can also model the problem with criterion (4), in which the number of double covered demands should be maximized. Nevertheless the constraints (12) must be replaced by constraints (16):

$$
\sum_{i \in I} a_{i j} y_{i} \geq 1+x_{j} \text { for } j \in J
$$

Concerning the solving technique for the problems described by the above presented models, it can be noted that all of them belong to the family of integer programming problems, more precisely zero-one integer programming problems and can be theoretically solved by any commercial solver, which contains some general integer programming algorithm, e.g. the branch and bound method, the cutting plane method or the branch and cut method. These general algorithms are able to solve to optimality real-sized covering problems, but only small instants of the allocation problems. To solve the problems with the criteria (1) or (2), we can make use a similarity between the problem (5)-(10) and the uncapacitated facility location problem [3]. The problem can be solved by the approach reported in [4] or [9], where a Lagrangean multiplier is introduced for the constraint (8) to relax it from the set of constraints. Then the problem takes a form of the uncapacitated facility location problem. To solve it, the procedure BBDual [9] was designed and implemented based on the principle presented in [3], which is the branch and bound method with special methods for obtaining of the lower bound. The procedure was embedded into the dichotomy algorithm, which was used to find a fitting value of the Lagrangean multiplier.

\section{Preliminary numerical experiments and criteria comparison}

We performed the numerical experiments with the data originating at the Slovak road network with 2916 dwelling places, which represent aggregations of potential patients. In this study, the electronic road map of Slovak Republic was employed. The numbers of inhabitants of dwelling places were known together with other attributes of the nodes. The current proposal of the emergency medical vehicle location consists of 264 places, but 41 of them duplicate or triplicate locations at some bigger cities and they have no influence on the studied accessibility in accordance to criteria (1), (2) and (3) considering the fact that these towns are represented by one node each. Based on this reduction, the 223 points (locations) were taken into consideration as the value $p$ in the primary problem. The sum of unallocated ambulances from the primary problem solution and the 41 multiple locations enter as value $p$ the secondary problem. These data enable to calculate the suggested criteria for the given scenarios of the vehicle speeds connected with the individual link classes. The considered speed scenario was $v=\langle 105,95,75,60,50\rangle$, which are assumed average speeds in kilometer per hour on highways, roads of first, second and third class and on the local roads respectively. The set of candidate locations was formed from all towns and villages with more than 300 inhabitants and present ambulance locations. This way, a set of 2284 candidate locations was obtained.

We solved all the above formulated problems for $T^{\max }=15$ minutes, whenever this limit was included into the model. In accordance to the type of criterion we employed the special algorithm BBDual or the general optimisation software Xpress- $M P$, if possible with respect to the size and structure of the associated model. The associated algorithms were run on a personal computer equipped with the Intel Core 26700 processor with parameters: $2.66 \mathrm{GHz}$ and $3 \mathrm{~GB}$ RAM.

The results of numerical experiments are reported in Table 1 where each row corresponds to one instance of the problem, which is specified by the used criterion and problem type ( $p$-primary or s-secondary). The row contains the objective function value (Objective) of the optimal solution, the computation time in seconds (Time $[\mathrm{s}]$ ) and the number of located ambulances (Loc). These figures are placed in the section BBDual or Xpress-MP in accordance to the used solution technique.

It turned out that the problem with criterion (2) was insolvable due to either huge time consumption or model size by both the 
approaches. That is why the optimal solution of only problems with criteria (1), (3) and (4) are reported in Table 1.

Table 1

\begin{tabular}{|c|c|c|c|c|c|c|c|}
\hline \multirow{2}{*}{ Criterion } & \multirow{2}{*}{ Type } & \multicolumn{3}{|c|}{ BBDual } & \multicolumn{3}{c|}{ Xpress-MP } \\
\cline { 3 - 8 } & & Objective & $\begin{array}{c}\text { Time } \\
{[\mathrm{s}]}\end{array}$ & Loc & Objective & $\begin{array}{c}\text { Time } \\
{[\mathrm{s}]}\end{array}$ & Loc \\
\hline$(1)$ & $\mathrm{p}$ & 13771837 & 3436 & 222 & - & - & - \\
\hline$(1)$ & $\mathrm{s}$ & 44203257 & 3574 & 42 & - & - & - \\
\hline$(3)$ & $\mathrm{p}$ & & & & 91 & 0.1 & 198 \\
\hline$(3)$ & $\mathrm{s}$ & & & & 286180 & 1.4 & 66 \\
\hline$(4)$ & $\mathrm{p}$ & - & - & - & 11684 & 0.3 & 264 \\
\hline
\end{tabular}

The comprehensive solutions were obtained from the primary and secondary solutions by simple addition of the zero-one resulting vectors $y^{p}$ and $y^{s}$. So in the comprehensive solution, 264 ambulances are deployed. The subscript $i$ of the nonzero allocation variable $z_{i j}$ for criterion (1) was obtained for each $j$ so that the equation (17) holds.

$$
t_{i j}(v)=\min \left\{t_{k j}(v): k \in I, 1 \leq y_{k}^{p}+y_{k}^{s}\right\}
$$

The value of variable $x_{j}$ for criteria (3) and (4) was obtained for each $j$ in accordance to the equation (18) or (19) respectively.

$$
\begin{aligned}
& x_{j}=\min \left\{1, \sum_{i \in I} a_{i j}\left(y_{i}^{p}+y_{i}^{s}\right)\right\} \\
& x_{j}=\max \left\{0, \min \left\{1, \sum_{i \in I} a_{i j}\left(y_{i}^{p}+y_{i}^{s}\right)-1\right\}\right\}
\end{aligned}
$$

This way, comprehensive solutions $B B D$ ual(1), Xpress-MP(3) and $X$ press- $M P(4)$ were obtained. Then the values of criteria (1)-(4) were computed for the solutions and these results are reported in Table 2.
Table 2

\begin{tabular}{|l|c|c|c|c|}
\hline & Criter. (1) & Criter. (2) & Criter. (3) & Criter. (4) \\
\hline BBDual(1) & 13677537 & 35109 & 15613 & 285324 \\
\hline Xpress-MP(3) & 23613635 & 182 & 91 & 188937 \\
\hline Xpress-MP(4) & 25597594 & 182 & 91 & 11684 \\
\hline Man-made & 16378985 & 92032 & 31672 & 431415 \\
\hline
\end{tabular}

The row Man-made in Table 2 corresponds to the current distribution of ambulance vehicles over the area of the Slovak Republic.

\section{Conclusions}

We presented four models of the medical emergency system design problem which are based on a different quality criterion. These quality criteria reflect possible approaches to the original problem with a general objective formulated as: to provide the best service to all inhabitants of a considered region. As any sophisticated designing process of real service system needs methods, which are able to provide it with a concrete solution in a sensible time, we tried to assign to these particular problem formulations some solving algorithms and performed preliminary computational experiments to verify suitability of the algorithms. With exception of the second criterion, we found that instances of the particular problem types were solvable in reasonable time. Furthermore, we compared the obtained results with the current structure of the medical emergency system of the Slovak Republic. We proved that the studied approaches could considerably improve the current system in all the considered objectives.

\section{Acknowledgment:}

This work was supported by grant VEGA 1/3775/06 and 6FP project RIPCORD-ISEREST.

\section{References}

[1] CURRENT, J., DASKIN, M., SCHILliNG, D.: Discrete Network Location Models, Drezner, Zvi (ed.) et al. Facility location. Applications and theory, Berlin: Springer, 2002, pp. 81-118

[2] DOERNER, K., F.; GUTJAHR, W., J.; HARTL, R., F.; KARALL, M.; REIMANN, M.: Heuristic Solution of an Extended DoubleCoverage A mbulance Location Problem for A ustria, Central European Journal of Operations Research, 4/2005, pp. 325-340

[3] ERLENKOTTER, D.: A Dual-Based Procedure for Uncapacitated Facility Location, Operations Research, 6/1978, pp. 992-1009

[4] JANACEK, J.: Service System Design in the Public and Private Sectors, Proc. of the International Conference "Quantitative Methods in Economics (Multiple Criteria Decision Making XII)“, June, 2004, Virt, ISBN 80-8078-012-9, pp. 101-108

[5] JANACEK, J.: The Medical Emergency Service System Design, Advances in Transport Systems Telematics, ed. Mikulski, J., Katowice, 2006, pp. 443-449

[6] JANACEK, J.: Time Accessibility and Public Service Systems, Proc. of the conference "Quantitative Methods in Economics", Bratislava, December, 2006, pp. 57-63

[7] JANACEK, J.: Safety on Roads from View of Emergency System Design, Journal of Information, Control and Management Systems, No 2/1, 2006, pp. 91-101

[8] JANACEK, J.: Evaluation Criteria for Emergency Service Stations Locations (in Czech), Sborník prispevku konference Infotrans 2007, September 2007, pp. 125-130 
[9] JANACEK, J., BUZNA L.: Facility Location in Distribution Systems, EDIS Zilina University Publisher, Zilina, 2007, 142 p.

[10] JANACKOVA M., SZENDREYOVA, A.: An Impact of Set of Centers of Distribution System design problem on computational time of solving algorithm, Advances in Transport Systems Telematics, ed. Mikulski, J., Katowice, 2006, pp. 109-112

[11] JANOSIKOVA, L: Optimal Location of Emergency Medical Service Stations in Term of Transportation Accessibility (in Slovak), Sbornik prispevku konference Infotrans 2007, September 2007, pp. 143-148

[12] JANOSIKOVA, L. Emergency Medical Service Planning, Communications - Scientific Letters of the University of Zilina, 2/2007, pp. 64-68

[13] MARIANOV, V.; SERRA, D.: Location Problems in the Public Sector, Drezner, Zvi (ed.) et al. Facility location, Applications and theory, Berlin: Springer, 2002, pp. 119-150. 